\title{
Lexis
}

Journal in English Lexicology

$8 \mid 2014$

Metaphor Studies in the English Language

\section{Reflets d'actualité/s : la métaphore politique et économique à l'interface du contexte international}

\section{Cathy Parc}

\section{(2) OpenEdition}

\section{Journals}

Édition électronique

URL : http://journals.openedition.org/lexis/234

DOI : 10.4000/lexis.234

ISSN : 1951-6215

Éditeur

Université Jean Moulin - Lyon 3

Référence électronique

Cathy Parc, «Reflets d'actualité/s : la métaphore politique et économique à l'interface du contexte international », Lexis [En ligne], 8 | 2014, mis en ligne le 09 janvier 2014, consulté le 10 décembre 2020. URL : http://journals.openedition.org/lexis/234 ; DOI : https://doi.org/10.4000/lexis.234

Ce document a été généré automatiquement le 10 décembre 2020.

\section{(c) (i) (9)}

Lexis is licensed under a Creative Commons Attribution-NonCommercial-NoDerivatives 4.0 International License. 


\title{
Reflets d'actualité/s : la métaphore politique et économique à l'interface du contexte international
}

\author{
Cathy Parc
}

"Still, context is everything " peut-on lire au détour de l'article «US economy Gains Steam as 200,000 Jobs Are Added» paru le 6 janvier 2012 dans le New York Times. Cette affirmation qui résonne comme un mot d'ordre herméneutique, invite le lecteur à considérer les faits dans leur contexte propre, au gré d'une appréhension holistique, et non de façon réductrice, uniquement pour eux-mêmes. Dans quelle mesure le contexte, dont l'étymologie indique qu'il constitue "un assemblage», notamment de fils avec lesquels tisser ${ }^{1}$, influe-t-il sur l'émergence de métaphores en prise directe avec l'actualité tant politique qu'économique dans les articles de la presse britannique ou américaine? Tenter de répondre ici à cette question centrale supposera de focaliser l'attention sur ces motifs du tissu stylistique où les sens s'entrecroisent, se nouent, pour faire figure et mieux refléter aux yeux du récepteur les effets chatoyants du discours. Effets spéculaires où le figuré renvoie au littéral l'image tout à la fois du même et de l'autre de sorte que l'interprétation n'a lieu d'être que dans un entre-deux doublement signifiant. Dans ce miroir que nous tend le journaliste, où l'objectivité supposée des faits le dispute à la subjectivité avérée de leur présentation, la trame de la situation internationale en général, et des événements récurrents comme les Jeux olympiques en particulier, permettra de mettre en relief les idiomatismes révélateurs de spécificités culturelles. Les comparaisons avec des coupures de presse françaises révéleront les procédés de surimpression entre le concret et l'abstrait qui démultiplient le sens au rythme de tournures propres au génie de chaque langue. À l'intérieur du cadre théorique défini par la linguistique cognitive et son approche de la métaphore conceptuelle, le fil directeur pointera vers les invariants auxquels les métaphores tant textuelles que visuelles donnent corps en les faisant s'incarner dans l'image, poinçon du style de l'auteur ou du dessinateur qui cherche à évoquer les faits plus qu'à les dire, pour mieux les faire parler. Dans cet exercice de ventriloquie où les lieux communs sont partagés, un même thème se 
prêtera à des variations originales qui nous feront donc entendre mais aussi voir l'un des contrepoints de la littéralité.

L'influence du contexte immédiat sur l'émergence de métaphores journalistiques se perçoit d'emblée à l'occasion d'événements internationaux marquants comme les Jeux Olympiques $^{2}$ : de nombreux articles ${ }^{3}$ tirent parti de l'engouement suscité par ces grandes manifestations sportives pour opérer un détournement d'attention vers les domaines de la politique et de l'économie ${ }^{4}$ où le jeu des différentes théories et de leurs mises en pratiques ne va pas non plus sans enjeux. La juxtaposition des deux contextes a priori bien distincts fait passer au premier plan l'idée-force de la compétition entre les pays, compétition à laquelle elle confère une dimension ludique comme pour rendre la lecture plus vivante, plus attrayante ${ }^{5}$. Parmi les procédés utilisés pour créer des interférences signifiantes, dont le bien-fondé se justifie par des visées pragmatiques, la métaphore est sans doute celui qui propage le plus rapidement et le plus efficacement l'onde de sens auprès du récepteur. Notamment la métaphore iconographique du dessin de presse qui fonctionne à la manière d'un déflecteur en illustrant la problématique tout en la présentant instantanément sous un autre angle, l'angle de son autre auquel la lient différences et similitudes. À l'immédiateté inhérente au support visuel s'ajoutent l'unicité de ce dernier et son gage d'originalité, qui donnent à cette métaphore émergente un caractère d'autant plus frappant qu'elles en font un singleton : contrairement à certaines métaphores linguistiques en effet, dont celle du Tour de France pour les articles de la presse économique française ${ }^{6}$, elle n'a pas vocation à réapparaitre ailleurs ${ }^{7}$. La photographie en exergue à l'article de Stephanie Flanders, rédactrice-en-chef du service économique de la $\mathrm{BBC}$, intitulé "Could the US economy go the Way of Japan?» (bbc.co.uk, 31 août 2011) en est un exemple probant:

(1) Is the US economy on the right track to recovery?

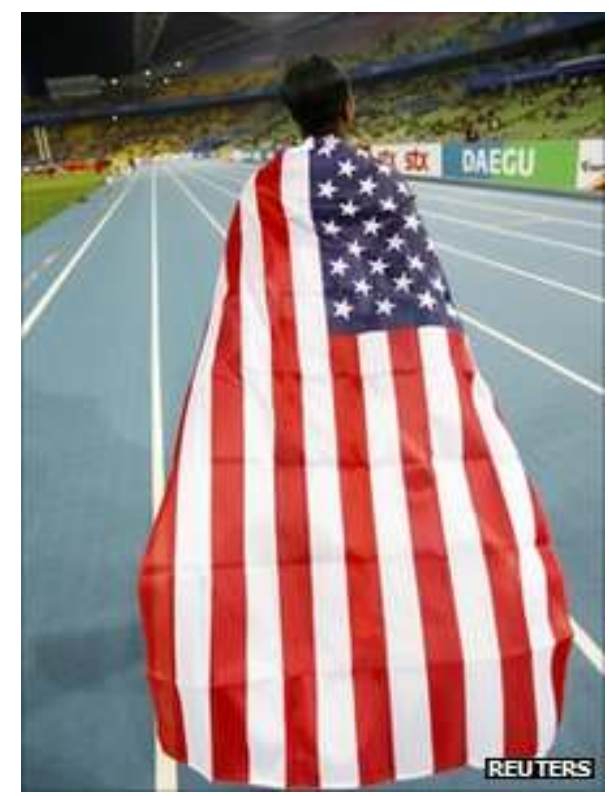

Le contexte premier des Jeux Olympiques dans lequel elle a été prise constitue le domaine-source choisi pour représenter le domaine-cible auquel est assimilée l'économie américaine $^{8}$. Or, cette photographie n'est pas métaphorique en soi, elle ne le devient qu'en raison du lien qu'elle entretient avec le titre de l'article et avec sa propre légende, "Is the US economy on the right track to recovery?». Seule la lecture de ces deux 
questions en écho, qui encadrent le cliché comme pour mieux nous en faire mesurer la portée, provoque une interprétation dans un sens figuré : les mots nous guident en déterminant à quel degré nous devons comprendre la mise en image qui les traverse certes mais les transcende aussi. De fait, la substitution du substantif spécifique « track » au substantif générique "way" semble justifier à elle seule l'accompagnement visuel dont les mécanismes sémiotiques dépendent de la métaphore conceptuelle TIME IS SPACE${ }^{9}$, TIME IS HORIZONTAL ${ }^{10}$, et de son corrélat par rapport métonymique HISTORY IS SPACE, l'histoire s'inscrivant elle-même dans le temps. Cette spatialisation temporelle se décline en métaphores secondaires qui mettent en perspective littéralement et figurativement l'évolution économique des États-Unis à travers le parallèle avec le Japon, visuellement matérialisé par les couloirs d'athlétisme, image que l'on retrouve également dans des articles de la presse française ${ }^{11}$ :

The economy of a country goes a certain way.

A track is a certain kind of way.

So the economy of a country goes on a certain track.

There are the right track to recovery and the wrong one.

The wrong one is Japan's.

So is the US on the right track?

3 Les correspondances entre les deux domaines mentionnés ${ }^{12}$, le sport en général, la course d'athlétisme en particulier, et l'économie, qui paraissent être les résultantes de certains des constituants de la métaphore de la structure événementielle - CHANGES ARE MOVEMENTS, ACTION IS SELF-PROPELLED MOTION, PURPOSES ARE DESTINATIONS, MEANS ARE PATHS, LONG-TERM, PURPOSEFUL ACTIVITIES ARE JOURNEYS ${ }^{13}$ - sont les suivantes :

\begin{tabular}{|c|c|c|}
\hline le stade & $\rightarrow$ & le monde \\
\hline l'athlète d'un pays & $\rightarrow$ & l'économie d'un pays \\
\hline l'allure de l'athlète d'un pays & $\rightarrow$ & le rythme de l'économie d'un pays \\
\hline $\begin{array}{l}\text { le couloir réservé à l'athlète d'un } \\
\text { pays }\end{array}$ & $\rightarrow$ & le destin économique d'un pays \\
\hline $\begin{array}{l}\text { la course = la compétition entre les } \\
\text { athlètes }\end{array}$ & $\rightarrow$ & $\begin{array}{l}\text { la course }{ }^{14}=\text { la compétition entre les économies des } \\
\text { différents pays }\end{array}$ \\
\hline $\begin{array}{l}\text { le couloir de l'un des athlètes les } \\
\text { moins performants }\end{array}$ & $\rightarrow$ & l'économie du Japon \\
\hline le couloir du meilleur athlète & $\rightarrow$ & $\begin{array}{l}\text { les meilleures politiques mises en œuvre pour } \\
\text { relancer l'économie }\end{array}$ \\
\hline $\begin{array}{l}\text { l'athlète le plus performant est le } \\
\text { plus rapide }\end{array}$ & $\rightarrow$ & $\begin{array}{l}\text { l'économie la plus performante est celle qui a le plus } \\
\text { fort taux de croissance }\end{array}$ \\
\hline & & \\
\hline
\end{tabular}


l'athlète qui passe la ligne d'arrivée le premier remporte la course le pays dont l'économie a le plus fort taux de croissance est la première superpuissance du monde photographiée de dos, elle se résume à une silhouette dont l'abstraction est accentuée par la symbolique du drapeau américain ${ }^{15}$. D'un point de vue visuel, cet artefact humain modifie les proportions du corps qui, bien qu'il occupe le premier plan et s'étende quasiment sur toute la hauteur du document, s'en trouve presque complètement occulté. D'un point de vue herméneutique, cet emblème national où se cristallise le sentiment patriotique et qui s'élabore également sur une relation synecdochique de la partie au tout (les étoiles $\rightarrow$ les états de l'Union, les treize bandes $\rightarrow$ les treize premiers états de l'Union, le drapeau $\rightarrow$ le pays) met en évidence l'ambivalence de l'incarnation ${ }^{16}$ qui sous-tend la métaphore. Concrète, la femme de couleur qui représente l'Amérique dans sa dimension économique au même titre que le Président Obama la représente dans sa dimension politique actuelle tandis que l'Oncle Sam en demeure la figure tutélaire d'une décennie à l'autre, n'en disparaît pas moins sous le poids des signifiés qui font écran au sens premier. À la surface de ce support à deux dimensions initialement anodin, auquel les règles de la perspective confèrent une profondeur illusoire, se projette une réflexion eschatologique sur le devenir économique des États-Unis et le destin de la nation tout entière, au gré d'une oscillation marquée entre le fatalisme suggéré par le titre et l'optimisme relatif qui caractérise la légende. L'espace de cette hésitation aura donc été comblé par la métaphore visuelle qui en synthétise dans l'instant, en silence, les tenants et les aboutissants pour tracer l'esquisse d'une réponse que les mots du texte développeront ensuite dans leur succession linéaire, et ce, dès les premières lignes :

There have been times, in the past few years, when the US policy makers weren't sure how the financial crisis was going to play out.

But on one point they were absolutely certain: the US would not - could not - go the way of Japan.

The economy was too flexible, the policy response to the crisis too dramatic, and the electorate too downright demanding for Japan-style stagnation to happen in the US.

Dans le cadre du détournement de l'image sportive à des fins d'argumentation économique, il est intéressant d'établir un contraste entre la photographie et le dessin de presse. À cet égard, l'illustration en couleurs de Dave Simonds pour l'article «Jamaica at 50 » de l'édition papier du numéro de The Economist du 21 juillet 2012 offre un terme de comparaison particulièrement révélateur : 


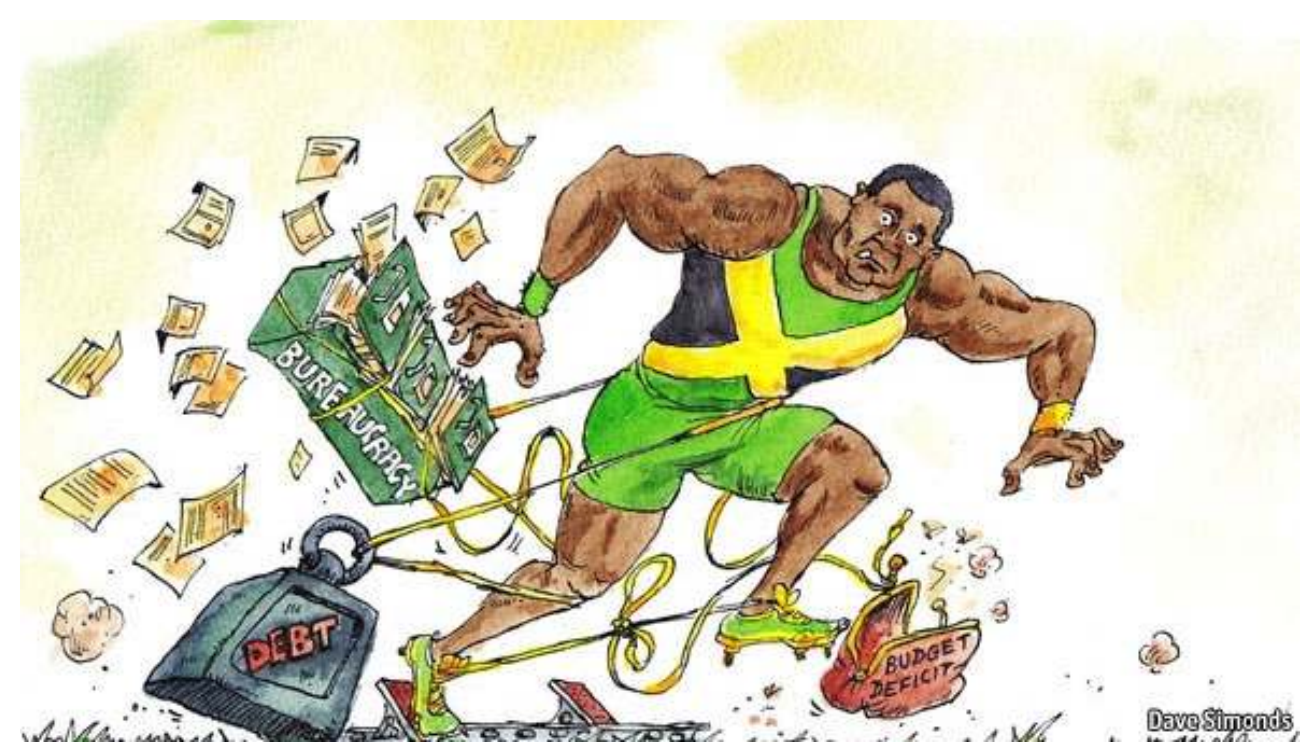

6 Cette fois-ci, l'élément iconographique est métaphorique en soi puisque même l'absence de titre et de sous-titre n'empêcherait pas le lecteur de le percevoir comme tel. De plus, il donne les clés de son interprétation car au sens littéral de la course d'athlétisme se superpose aussitôt le sens figuré dénoté par l'incipit et par les mots en majuscules qui font intrusion dans le support visuel. Les substantifs BUREAUCRACY, DEBT, BUDGET DEFICIT témoignent de la manipulation didactique à laquelle le dessinateur soumet son moyen d'expression. La photographie montrait de façon plus objective un pan de la réalité authentique, que des vocables extrinsèques transplantaient dans un contexte avec lequel certaines ressemblances le faisaient entrer en résonance pour en définir la portée. Ce dessin-ci privilégie, lui, l'explicitation intrinsèque tout en entretenant un flottement intentionnel entre le réel et l'imaginaire : passé au prisme de la subjectivité de l'artiste, il trace des lignes et traits d'humour pour composer, dans la dualité, une vision personnelle ${ }^{17}$ de la situation économique en Jamaïque. La métaphore centrale, qui se constitue à nouveau à partir d'une personnification de la patrie dont participe le drapeau national, même si elle est ici masculine, s'articule non seulement autour des similitudes entre les domaines économique et sportif mais surtout autour des différences qui les opposent. Si les athlètes jamaïquains sont habituellement réputés pour leurs excellents résultats aux Jeux Olympiques, il semble en aller tout autrement de l'économie de leur pays. Effectivement, leurs performances respectives sont inversement proportionnelles, comme l'indiquent clairement les cinq premières lignes du texte :

THE world is used to trailing behind Jamaican sprinters. The small island has won a string of world records, and may claim more at the 2012 Olympics.

Its economy, however, is not so speedy : on current forecasts it will finish the year with the slowest average growth rate since 2000 in the Americas - behind even earthquake-stricken Haiti. On August 6th Jamaica will celebrate the 50th anniversary of its independence. But the festivities will be muted by frustration with its performance.

7 Ce qui paraît d'autant plus paradoxal que la Jamaïque dispose de nombreux atouts :

The Jamaican economy should by rights be booming. The island is just a 90-minute flight away from the United States, the world's biggest market, with which it shares a language. It is on the shipping route to the Panama Canal, and has a spacious 
natural harbour in Kingston. It is politically stable, without the ethnic tensions that have riven other Caribbean nations.

C'est donc à l'effet de surprise qui se trouve répercuté sur le lecteur que la métaphore doit son efficace: la formule d'usage prononcée pour donner le départ d'une course est remplacée par « On your marks, get set, ... oh » où les points de suspension matérialisent dans la langue le retard pris par le sportif, tandis que le jeu de mots sur la paronomase entre le verbe à l'impératif « go » et l'interjection « oh » traduit avec réalisme la réaction tant du coureur, dont le visage, notamment le regard, est particulièrement expressif, que de la personne qui donne le signal et des spectateurs. Le second sous-titre joue un rôle tant d'encodage de la métaphore linguistique que de décodage de la métaphore visuelle qui suit puisqu'il développe l'analyse des correspondances entre littéral et figuré que cette dernière synthétise :

\begin{tabular}{|c|c|c|}
\hline l'athlète jamaïquain & $\rightarrow$ & l'économie de la Jamaïque \\
\hline la course & $\rightarrow$ & $\begin{array}{l}\text { la compétition entre les économies de différents } \\
\text { pays, notamment aux Amériques }\end{array}$ \\
\hline les starting blocks & $\rightarrow$ & $\begin{array}{l}\text { l'indépendance de la Jamaïque par rapport à la } \\
\text { Grande-Bretagne }\end{array}$ \\
\hline avoir des difficultés & $\rightarrow$ & se débattre \\
\hline sortir des starting blocks & $\rightarrow$ & croître $^{18}$ \\
\hline rester dans les starting blocks & $\rightarrow$ & ne pas croître \\
\hline $\begin{array}{l}\text { se faire dépasser par tous les autres } \\
\text { concurrents et terminer dernier }\end{array}$ & $\rightarrow$ & avoir le taux de croissance le plus bas \\
\hline
\end{tabular}

9 De plus, Dave Simonds a fait le choix d'introduire des métaphores dans la métaphore première en vertu d'une mise en abyme qui établit une équivalence entre le portemonnaie vide et le déficit budgétaire, entre le poids en fonte ou en laiton et la dette nationale écrasante, entre les tiroirs métalliques d'où s'échappent de nombreuses feuilles de papier et la bureaucratie excessive des administrations nationales. Ces métaphores secondaires disent dans la conjonction des étiquetages non figuratifs qui les désignent pour ce qu'elles sont, ce que le dessin seul aurait, lui, laissé entendre dans le silence du figuratif. La désignation rend abstrait ce que l'objet rend concret, générant ainsi une tension entre les deux médias utilisés, moyens d'impression et d'expression dont les élans contradictoires se résolvent dans une conciliation tant méta-linguistique que métagraphique. Le lien de cause à effet conçu dans la linéarité de la représentation spatiale du temps est le domaine-cible dont les cordelettes nouées autour du corps de l'athlète décrivent le domaine-source, dont la fonction consiste à figurer implicitement les chaînes qui entravent la liberté de mouvement du sportif dès lors assimilé dans notre imagination à un prisonnier ${ }^{19}$. Si le dessinateur s'attache à respecter certaines des conventions artistiques d'usage, notamment celles de la bande dessinée, lorsqu'il évoque les efforts du personnage par de petits nuages de poussière soulevés dans l'air, c'est donc aussi pour 
mieux s'en détacher en surimposant une image virtuelle à celle qu'il dessine. Il nous indique par là-même que le signifié profond n'est pas représenté en soi mais se construit dans le regard complice que nous échangeons avec lui lors de l'opération de lecture que prolonge l'interprétation. Ainsi, un simple détail comme l'herbe qui tapisse le couloir d'athlétisme est un embrayeur d'imaginaire qui nous fait comprendre que la course n'est que le pré-texte à l'évocation de la Jamaïque en tant qu'entité économique et géographique, à travers trois des maux qui l'accablent et l'empêchent de parcourir quelque chemin que ce soit sur la voie de l'indépendance, si bien qu'au bout de dix ans, le pays se trouve toujours au point de départ.

10 Ce jeu entre présence et absence, entre l'image réelle qui métaphorise un aspect de la réalité et l'image virtuelle qu'elle suscite en nous et dont elle est l'un des signifiants, est poussé à l'extrême dans la photographie placée en tête de l'article de Mark Mardell, rédacteur-en-chef de la BBC pour l'Amérique du Nord, «Is the US caught in the slow lane? »(bbc.co.uk, 5 juillet 2012) :

(3) People living in bankrupt Stockton give their verdict on the US economy

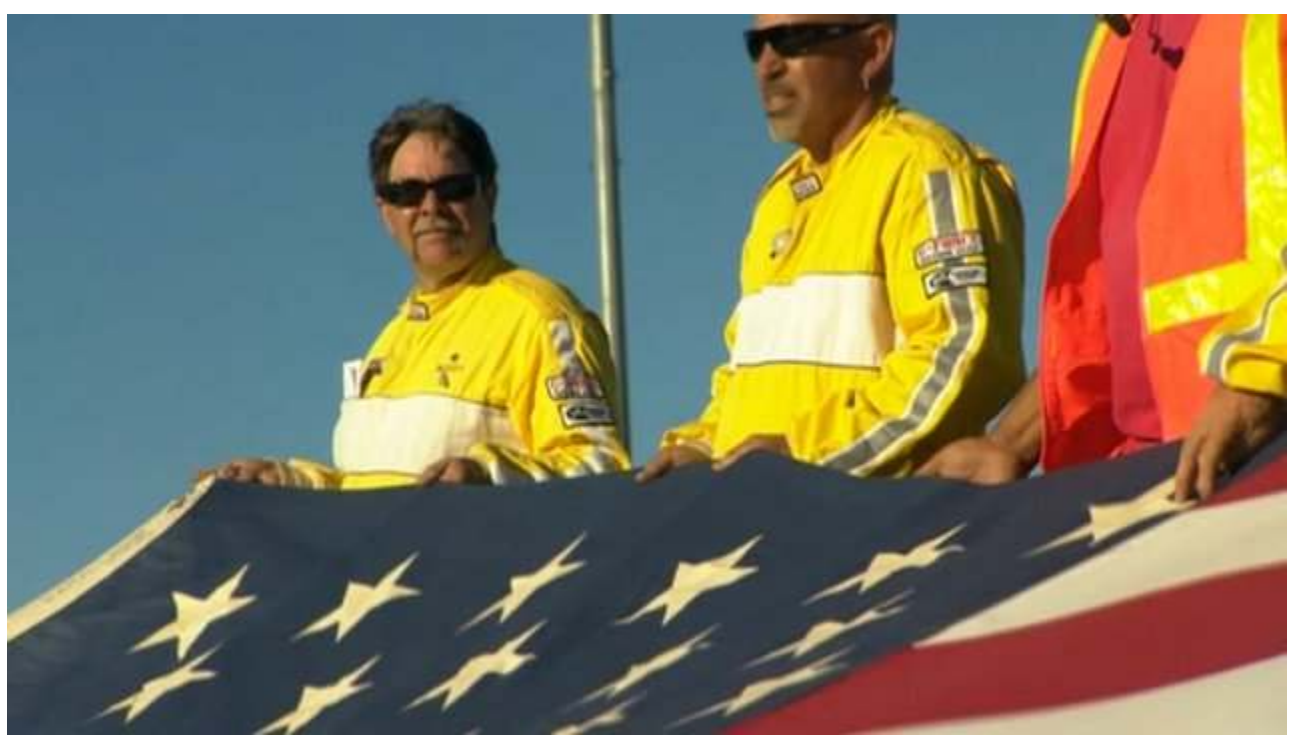

11 En effet, la voie pour véhicules lents, qui ressortit de la réalité quotidienne et non de l'environnement sportif dans lequel elle se trouve ici transplantée, n'y est nullement visible, de sorte que la métaphore visuelle paraît doublement in absentia. Il est donc plus difficile pour le lecteur d'établir immédiatement un rapprochement entre le titre et l'illustration, parce que le domaine-source des moyens de transport sur route ne s'y manifeste que par métonymie, voire par synecdoque, à travers le portrait en buste de trois coureurs ou techniciens automobiles qui tiennent dans leurs mains un coin du drapeau américain. Ce hiatus souligne le fait que l'illustration fonctionne davantage à la manière d'une amorce incitant le lecteur à prendre connaissance du texte pour en savoir davantage. Si les quatre points qui sont ensuite énumérés dans un style télégraphique pour communiquer l'essentiel du message semblent justifier la métaphore linguistique «US economic growth slows to $1.5 \%$; Bernanke says Libor system flawed; US retail sales fall back again; US Fed keeps policy options open »-, en revanche les quatre premières lignes de l'article éclairent le sens strictement littéral de la photographie :

At a California racetrack the souped-up cars that have been revving their way around the circuit stop and fall silent. 
Their roar is replaced by a deeply soulful rendition of the Star-Spangled Banner as a gigantic American flag is unfurled across the course as part of 4 July celebrations. comme le suggère la comparaison avec les photographies placées au début des articles «US economic growth slows in second quarter » (bbc.co.uk, 27 juillet 2012) et « Retail sales down in June for third straight month » (bbc.co.uk, 16 juillet 2012) :

(4) A slowdown in consumer spending contributed to the overall slowdown in economic growth

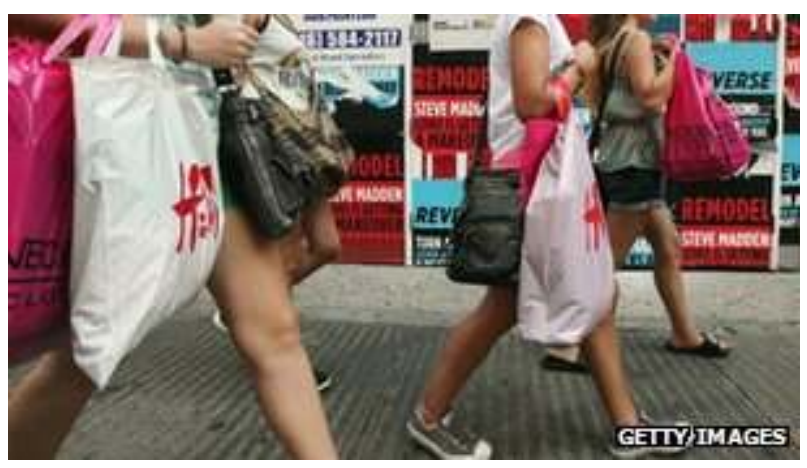

(5) Weak retail sales have raised fears over the strength of the economy

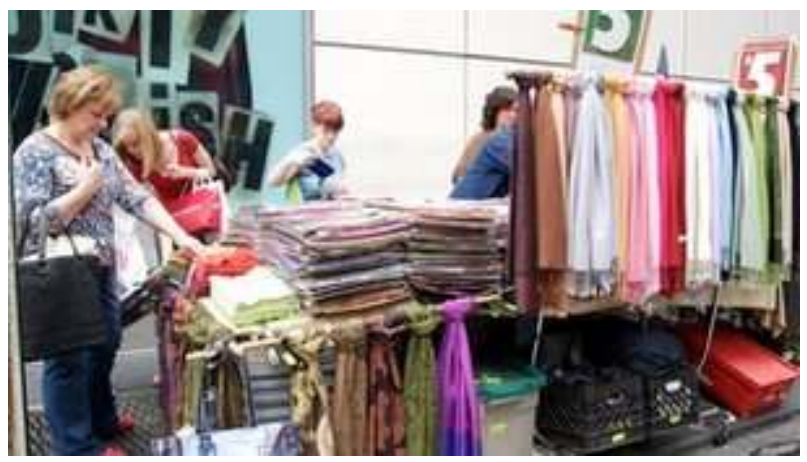

Sur la première, seul le buste et les jambes des passantes sont donnés à voir, ce qui accentue l'impression d'anonymat, d'impersonnalité dans un élan vers l'abstraction et l'universalisation qui vise à communiquer l'idée de mouvement tout en réduisant les personnes présentées à l'action stéréotypée de l'achat. La linéarité spatiale orientée de gauche à droite selon la vision occidentale ${ }^{21}$ métaphorise la linéarité temporelle inhérente à la succession du passé, du présent et de l'avenir, évoquée dès le titre à travers le groupe prépositionnel « in second quarter ", au gré des correspondances établies entre le pas des consommatrices et le taux de croissance économique américaine. Mais c'est à 
nouveau la relation métonymique de la partie au tout qui prédomine selon un principe de généralisation en s'articulant autour des équivalences suivantes :

\begin{tabular}{|l|l|l|}
\hline $\begin{array}{l}\text { les achats qui se trouvent dans les sacs } \\
\text { plastiques ce jour-là }\end{array}$ & $\rightarrow$ & $\begin{array}{l}\text { les achats effectués pendant la période } \\
\text { considérée (le second trimestre) }\end{array}$ \\
\hline $\begin{array}{l}\text { les achats effectués pendant la période } \\
\text { considérée }\end{array}$ & $\rightarrow$ & l'indice à la consommation aux États-Unis \\
\hline les consommatrices & $\rightarrow$ & tous les consommateurs américains \\
\hline les vitrines en arrière-plan & $\rightarrow$ & les boutiques correspondantes \\
\hline ces boutiques-là & $\rightarrow$ & tous les commerces du pays \\
\hline
\end{tabular}

Cette fois, c'est la légende «A slowdown in consumer spending contributed to the overall slowdown in economic growth » qui pousse le lecteur à reconsidérer l'image pour en modifier sa lecture initiale et en saisir, a posteriori, le sens métaphorique potentiel.

La photographie ou le dessin de presse se révélant plus ou moins métaphorique, il semble donc qu'il s'établisse un gradient entre les pôles que définissent la substitution immédiate du figuré au littéral et leur conjonction par surimpression simultanée ou différée. Si l'entrée en matière s'opère souvent de façon inversée dans les articles étudiés puisque les domaines du sport et de la vie quotidienne apparaissent comme premiers par rapport au domaine économique, elle suppose également l'introduction d'une dimension anthropomorphique et donne lieu à des "métaphores ontologiques " ${ }^{22}$ : l'économie d'un pays est décrite sous les traits d'un sprinteur aux Jeux Olympiques, d'un groupe de consommatrices, d'un patient en voie de guérison ou non, sous la forme d'une voiture de course ou d'un navire avec le président ou le chef du gouvernement à la barre. Mais, dans tous les cas, les éléments narratifs qui constituent chaque fois une intrigue sous-jacente particulière s'avèrent d'une importance capitale et imposent le romanesque comme clé de lecture et d'interprétation. Dans la collusion entre les genres du roman et du reportage sportif qui caractérise nombre de ces articles de la presse spécialisée, il s'agit de suivre la grande aventure des chiffres qui relate les tendances présentes et passées de la micro- et de la macro-économie tout en attribuant des caractéristiques humaines à ces phénomènes abstraits. En se fondant sur son expérience personnelle, sur celle d'un proche ou d'une connaissance, le lecteur peut ainsi se projeter d'autant plus facilement dans cette science qu'il est appelé à faire preuve d'empathie et à revivre la communion collective propre aux événements sportifs. Il s'investit émotionnellement ${ }^{23}$, donc subjectivement, dans une sphère théoriquement objective de sorte que les modalités herméneutiques ne sont plus les mêmes : des enjeux humains se profilent derrière les enjeux économiques qu'il n'est dès lors plus question d'aborder de manière froide, quasi clinique. Par conséquent, ce qui se joue dans l'instauration de ce nouveau contrat de lecture, c'est la (re)présentation de l'économie comme une science qui se veut aussi science humaine - jeu de la métaphore qui tire parti du jeu, de la marge, entre le littéral et le figuré, et jeu de l'actant voire de l'acteur qui en est l'incarnation vivante. Ainsi, lorsque le scénario sous-jacent correspond à celui de l'adaptation cinématographique d'un roman, le dessin de presse parait se confondre avec une photographie empruntée au film, alors même que ses lignes épurées le font tendre vers le dessin animé. Cette 
ambivalence caractérise le document iconographique qui accompagne l'article "The Spanish patient » paru dans The Economist le 28 juillet 2012 :

(6) The Spanish patient

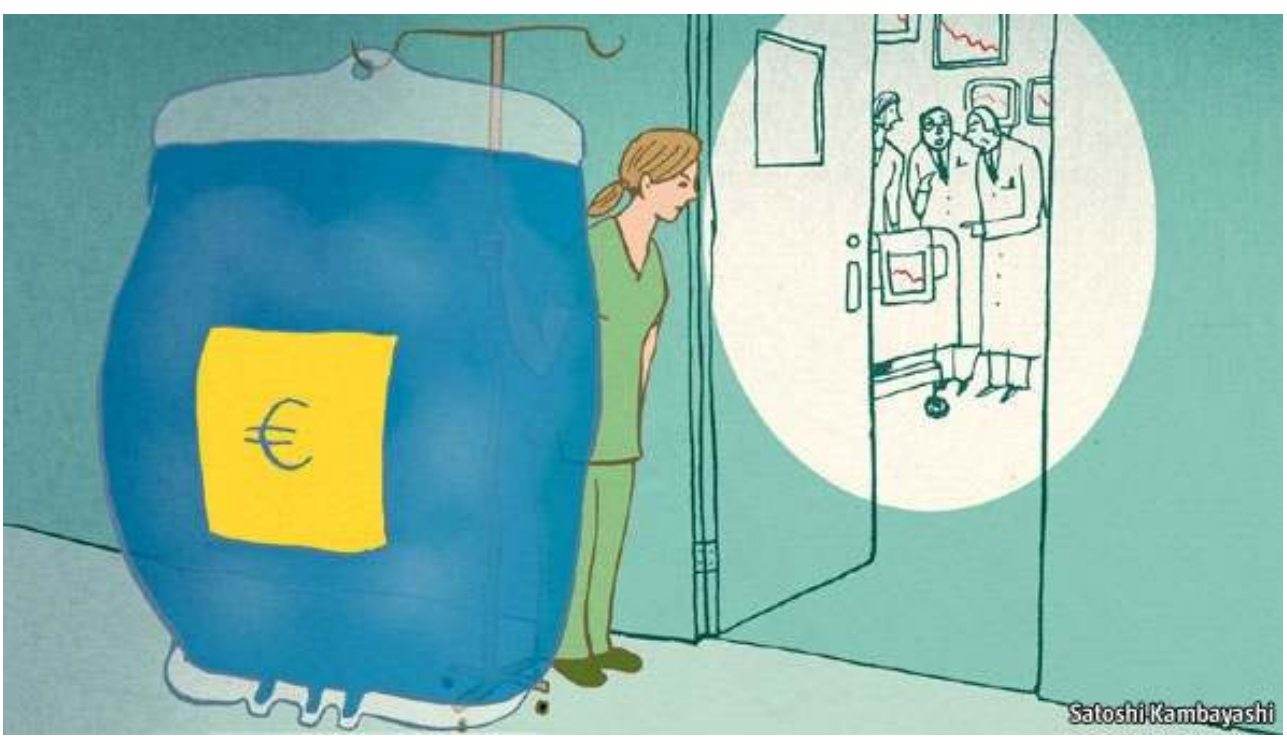

Pour quiconque a lu le roman The English Patient de Michael Ondaatje ou vu le film d'Anthony Minghella, le titre "The Spanish patient » suffit à lui seul à déclencher une mise en images, des scènes de l'œuvre fictionnelle ou filmique allant immanquablement lui revenir en mémoire. Dans un second temps, le dessin viendra prolonger les effets de cet embrayeur d'imaginaire en développant la formule de son intertextualité selon la métaphore le patient $\rightarrow$ le pays, le patient espagnol $\rightarrow$ l'Espagne, de sorte qu'il livrera lui aussi les clés de son interprétation. En corrélation directe avec le sous-titre «A full bailout of the euro area's fourth-largest economy is looming », le symbole de l'euro figurant sur ce qui est censé représenter la poche de sang prévue pour la transfusion, fait partie des correspondances métaphoriques articulées autour de la métaphore conceptuelle THE ECONOMY IS A BODY ${ }^{24}$ :

\begin{tabular}{|l|l|l|}
\hline l'euro & $\rightarrow$ & le sang \\
\hline la transfusion d'euros & $\rightarrow$ & la transfusion de sang \\
\hline $\begin{array}{l}\text { le maintien des fonctions vitales de la } \\
\text { quatrième économie européenne }\end{array}$ & $\rightarrow$ & $\begin{array}{l}\text { le maintien des fonctions vitales du } \\
\text { patient }\end{array}$ \\
\hline $\begin{array}{l}\text { or, le patient } \\
\text { les tracés des test médicaux effectués, visibles } \\
\text { au bas du lit et sur le mur de la chambre } \\
\text { d'hôpital }\end{array}$ & $\rightarrow$ & $\rightarrow \begin{array}{l}\text { l'Espagne } \\
\text { juger différents paramètres permettant de }\end{array}$ \\
\hline ces courbes descendantes du patient \\
\hline donc, la transfusion d'euros & $\rightarrow$ & $\begin{array}{l}\text { celles des différents indices permettant de } \\
\text { définir la situation économique espagnole }\end{array}$ \\
\hline
\end{tabular}




\begin{tabular}{|l|l|l|}
\hline l'infirmière & $\rightarrow$ & l'Europe \\
\hline les médecins & $\rightarrow$ & $\begin{array}{l}\text { les hommes politiques espagnols qui ont } \\
\text { pris les décisions jusque-là }\end{array}$ \\
\hline
\end{tabular}

L'élément le plus frappant, c'est que le patient lui-même n'est pas représenté, étant caché par la porte ouverte; il est donc in absentia puisqu'il est invisible au lecteur et in praesentia puisqu'il est au cœur des préoccupations des trois médecins qui sont rassemblés à son chevet pour débattre de son cas et dont l'infirmière surprend la conversation dans le couloir. Ce parti-pris de non-figuration qui fait passer au premier plan le traitement médical, c'est-à-dire les mesures économiques à prendre d'urgence pour sauver l'Espagne de la récession dans laquelle elle s'enfonce, accentue la dimension déontique du dessin, dont Line et Per Aage Brandt [2005: 245] donne cette définition générale: «deontic meanings indicate states of affairs that ought, or ought not, to be the case according to some principle that the speaker indirectly embodies or represents in the act of speaking ».

17 Cette tendance marquée à la personnification transparaît avec d'autant plus de force que nombre de graphiques filent la métaphore initiale, alors qu'ils correspondent théoriquement au pôle objectif de l'argumentation si leur mode de calcul ne peut être remis en cause. La prédominance du narratif sur le factuel s'impose comme une constante, sa fonction étant de contrer le côté parfois abscons, parfois rébarbatif de ces représentations abstraites. Le lecteur est ainsi invité à s'attarder plus longuement qu'à son habitude sur ces diagrammes qui lui permettent non seulement de visualiser des phénomènes économiques tangibles souvent non controversés, auxquels le texte vient de faire allusion, mais surtout de considérer un élément-clé de l'intrigue qui a été instaurée dès le titre. L'effet produit est comparable à celui qui résulterait de la comparaison entre les photographies d'un personnage de roman ou de film au fil du temps, comme le montrent clairement les tracés géométriques et les données chiffrées de l'illustration intitulée «Cardiac arrhythmia », qui vient ponctuer la dernière partie de l'article «The Spanish patient » : 


\section{Cardiac arrhythmia}

Ten-year government bond yields, \%

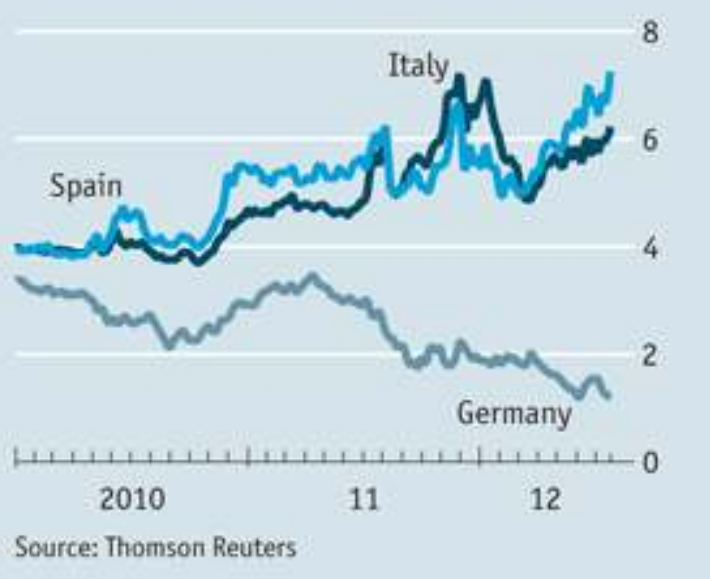

Immédiatement posé, le diagnostic médical ne génère une métaphore que dans sa juxtaposition avec la légende qui en définit defacto le domaine-cible («Ten-year government bond yields ») et détermine le sens premier tout en inversant les métaphores conceptuelles habituelles UP IS GOOD OR BETTER et DOWN IS BAD OR WORSE, étant donné que le terme négatif «arrhythmia» a été choisi comme critère pour la démonstration. Ce graphique financier se métamorphose alors en cardiogramme au gré des correspondances métaphoriques l'économie $\rightarrow$ le cœur, les courbes économiques $\rightarrow$ le tracé de l'arythmie du patient, et de la relation synecdochique entre les rendements des obligations de l'État espagnol et la situation économique en Espagne. Il modifie les modalités de notre lecture en suscitant des émotions, notamment notre compassion, de même que celui qui accompagne l'article «Jamaica at 50. On your marks, get set ...oh", dont il rappelle le titre et le dessin liminaire en en filant la métaphore :

(8) Off the pace

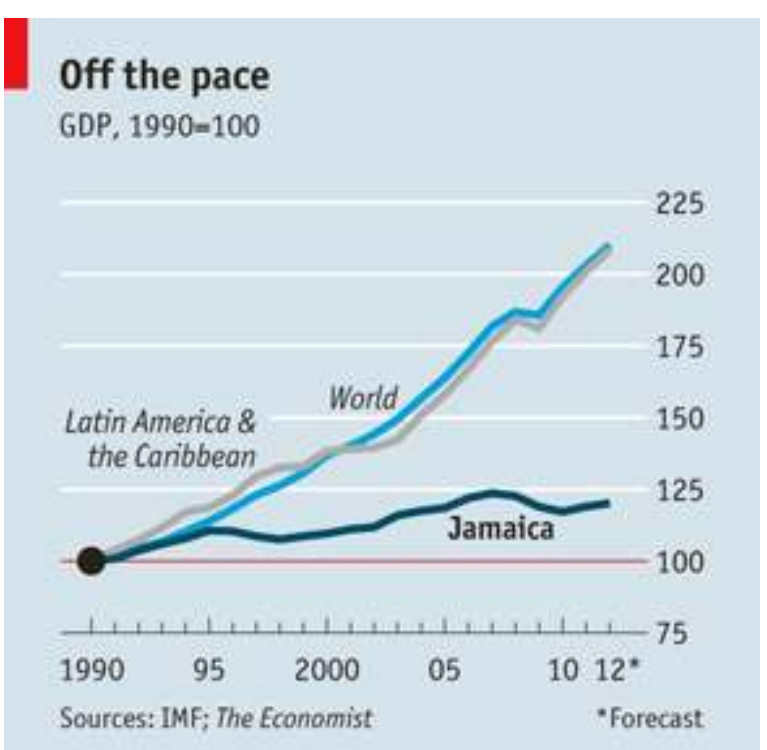


Il contribue de la sorte à la structure sous-jacente de l'argumentation au fil d'expressions comme «Jamaica has reasons for its plodding growth rate» (1. 21), "Jamaica has run fiscal deficits in 44 of its 50 years of independence » (1. 29), « The private sector has also been shackled by bureaucracy » (1. 44), et " Jamaica is due a spurt of growth» (1.40). Ces dernières font figure de jalons dans le raisonnement, de points de repère qui marquent la persistance de l'idée directrice initiale pour aboutir aux équivalents métaphoriques complémentaires :

\begin{tabular}{|l|l|l|}
\hline un sprinteur aux Jeux Olympiques & $\rightarrow$ & l'économie d'un pays \\
\hline l'allure d'un sprinteur & $\rightarrow$ & le taux de croissance économique d'un pays \\
\hline les autres sprinteurs & $\rightarrow$ & les économies des autres pays \\
\hline l'allure des autres sprinteurs & $\rightarrow$ & $\begin{array}{l}\text { le rythme de la croissance économique au } \\
\text { niveau mondial }\end{array}$ \\
\hline $\begin{array}{l}\text { l'allure du sprinteur jamaïquain est bien } \\
\text { plus lente }\end{array}$ & $\rightarrow$ & l'économie de la Jamaïque n'arrive pas à suivre \\
\hline
\end{tabular}

La distance parcourue sur le plat lors de la course aux Jeux Olympiques se trouve projetée sur l'axe vertical où l'écart creusé entre la Jamaïque et les autres pays d'Amérique latine ou des Caraïbes en particulier ainsi que du reste du monde en général, est d'autant plus manifeste qu'il est précisément chiffré. Se laissant également apprécier bien plus longuement que lors de l'événement sportif où il ne se calcule qu'en secondes ou en fractions de seconde, il peut être visualisé selon une ligne verticale qui semble reproduire le couloir d'athlétisme horizontal en perspective : le jeu de la projection géométrique est tel que le lecteur qui considère l'année 2012, date des derniers JO, a bel et bien l'illusion de percevoir les différents coureurs au moment de l'arrivée, plus qu'il ne garde à l'esprit la comparaison entre les produits intérieurs bruts des pays concernés. C'est sur un effet d'optique similaire que se fonde le diagramme épuré de l'article «U.S. Chose Better Path to Recovery » dont l'originalité procède de la référence intertextuelle au conte de Charles Dickens A Tale of Two Cities modulée dans la légende en A Tale of Two Continents et perpétuée à la ligne 15 ( No one factor made the difference in the divergent paths the two continents have taken. But there are two - both related to financial conditions - that were very important. In each case, it appears that the United States did a much better job »):

\section{(9) Graphic. A Tale of Two Continents}

Ce graphique est tellement synthétique que ni l'axe des abscisses ni celui des ordonnées n'ont été représentés ou encodés, de sorte qu'il serait illisible sans le titre, les sous-titres et les premières lignes du texte qui en éclairent le sens :

Now, the pictures appear very different. The unemployment rate in the Unites States has been steadily falling, while the unemployment rate in the euro zone has climbed to its highest level since the currency was introduced more than a decade ago. There is still some double-dip talk in the United States, but in many European countries it is a reality. 
En convoquant les connaissances partagées censées relever de la culture générale des lecteurs, le journaliste transpose dans le contexte d'une œuvre littéraire classique des données économiques dont la représentation sous forme mathématique est des plus sommaires, comme si elle était appelée à s'effacer autant que possible. Les chiffres en eux-mêmes n'importent pas, puisqu'ils ont été omis, seul semble compter l'effet visuel produit par ces deux courbes qui ne valent que par leur corrélation. Du fait de ce dépouillement intentionnel, le lecteur retient seulement l'image minimaliste d'un écart croissant même s'il ne peut l'évaluer précisément, aucun élément scientifique ne devant semble-t-il venir troubler son plaisir de lecture. Cette propension à la concrétude, que trahit un élan vers l'abstraction paradoxale de la littérature, caractérise aussi le graphique « Tracking US Growth » de l'article « Blow for Obama as new figures show U.S. economy sloping even further as Americans cut back on spending " paru le 27 juillet 2012 dans The Daily Mail. La toute dernière baisse du taux de croissance y est représentée par un segment descendant entre trois indices qui, en convoquant la métaphore linguistique de la pente - «Data from the Commerce Department shows how the economy is on a downward slope again after promising growth following the recession »- devient en soi métaphore visuelle :

(10) Tracking US Growth

Tracking U.S. Growth

Seasonally adjusted annual rate, in percent

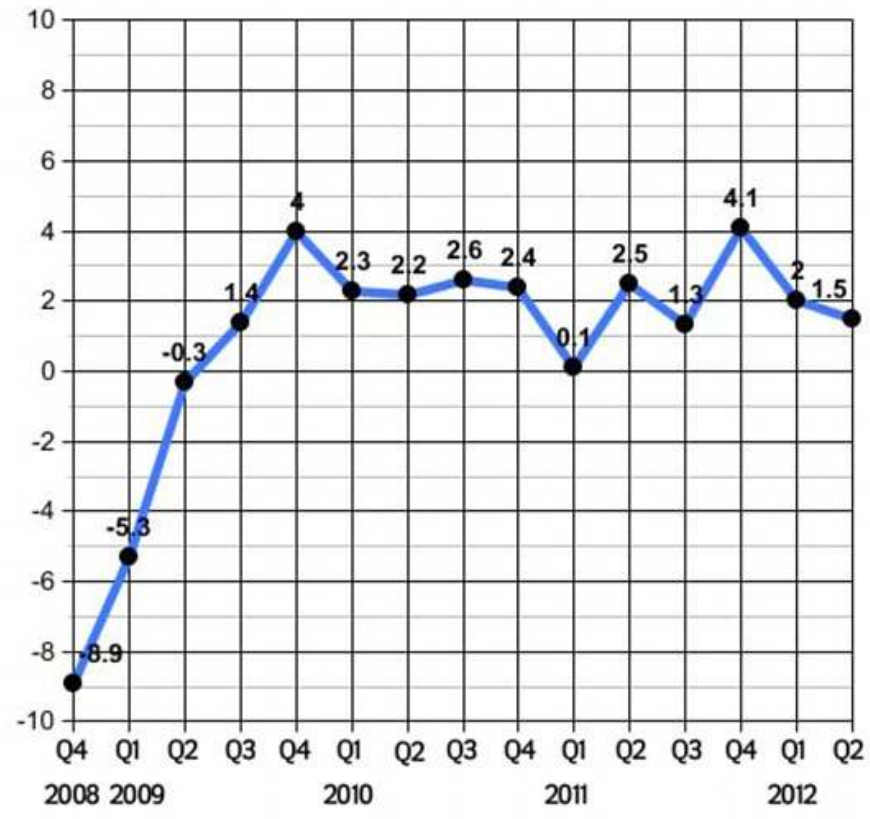

Source: Commerce Department

Il en va de même du graphique inclus au milieu de l'article « Made in Britain. Exports are growing, but too slowly to rescue the economy » (The Economist, 21 janvier 2012), dont la légende « Time to BRIC it? » est rédigée sous la forme d'une question elliptique purement rhétorique : 


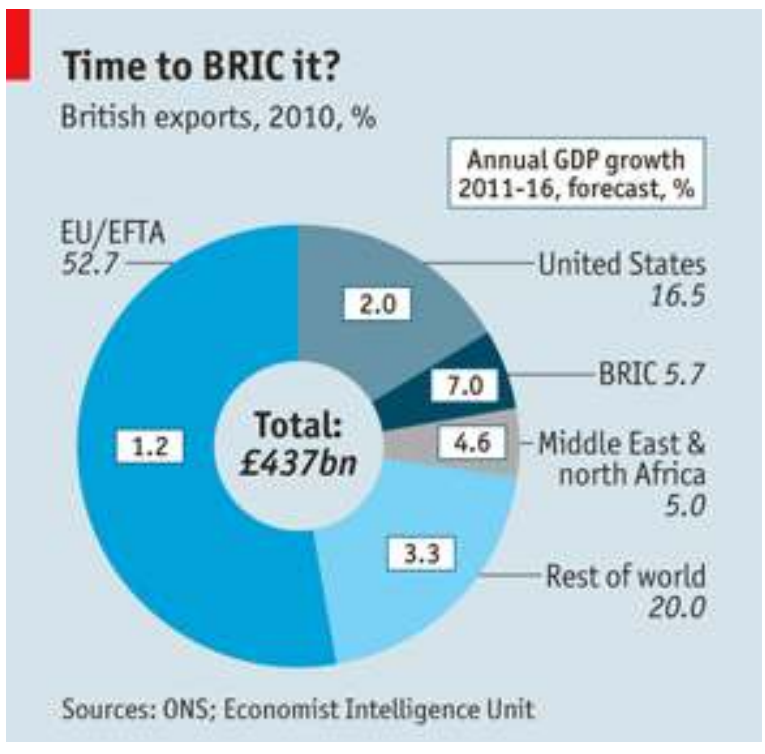

À la lecture des pourcentages, les prédictions à long terme font clairement apparaître que le bloc constitué par le Brésil, la Russie, l'Inde et la Chine connaîtra le plus fort taux de croissance du PIB entre 2011 et 2016, à savoir 7 \%, alors que ceux de l'Europe et des ÉtatsUnis seront respectivement de $1,2 \%$ et $2 \%$. La désignation synthétique "BRIC » s'explique par des raisons tant sémantiques qu'euphoniques: elle est obéit au même schéma de composition que les expressions définissant des moyens d'action ou des moyens de transport comme " to foot it ", « to hop it » etc., qui doivent notamment leur efficace pragmatique à l'emploi de monosyllabes. C'est pourquoi l'interrogation « Time to Brazil-Russia-India-China it?» qui est pourtant plus compréhensible, notamment si le lecteur n'est pas familier de l'acronyme ici utilisé comme verbe, n'aurait pas été concevable car elle ôte à l'énoncé son pouvoir de formule magique de la réussite économique. De plus, bien qu'aucune confusion ne soit possible avec le mot «brick ", puisque l'illustration est insérée dans un texte écrit, la paronomase est néanmoins à l'origine d'un jeu de $\operatorname{mots}^{25}:$ l'image des briques convoque à son tour celle de l'édifice en construction dont les qualités de solidité lui valent de devenir le prototype des édifices à consolider dès à présent ou à construire à l'avenir. Le caractère frappant de la formulation est la résultante de la métaphore linguistique ainsi forgée dans et par l'humour à partir de la relation synecdochique entre le tout et la partie, que l'illustration reprend dans sa nature même, le camembert étant divisé en portions qui représentent un pays ou un groupe de pays par l'indice économique qui lui est propre et par un pourcentage d'exportations britanniques dont il est destinataire. Cette lecture à deux niveaux accentue le contraste entre le bloc BRIC et les autres du fait que le premier est matérialisé non seulement par la plus petite portion du camembert, correspondant au plus faible volume d'exportations en provenance de la Grande-Bretagne, mais aussi par le plus fort taux de croissance à l'échelle nationale, signe d'une meilleure balance commerciale.

À l'exception de «The Spanish patient » qui file la métaphore initiale du début à la fin ${ }^{26}$, l'étude du corpus permet d'établir en constante le fait qu'il n'y ait pas de diktat de la

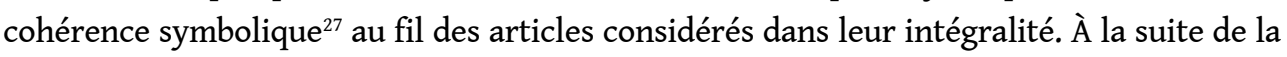
photographie ou du dessin de presse, le texte procède à l'approfondissement du champ de 
vision défini par la métaphore visuelle avec l'apport de données économiques chiffrées et de métaphores linguistiques empruntées à plusieurs domaines qui participent de la présentation des politiques mises en œuvre ainsi que de l'analyse de leurs tenants et aboutissants. L'esthétique du fragment prévaut au sein de ce patchwork où plusieurs intrigues se succèdent sans transition ni lien implicite ou explicite, rappelant en cela la technique du collage artistique au gré duquel les images sont autant de mini-vignettes valant tant pour elles-mêmes que pour l'effet produit par leur association hétéroclite. Ainsi, des passages traitant d'économie pure entièrement rédigés dans un style communément caractérisé comme étant littéral, alternent immanquablement avec des passages émaillés de métaphores dont le domaine-source emprunte à une multitude de champs lexicaux même si des invariants se dégagent, tels ceux des sports, des voies terrestres ou maritimes, des modes de transport, de la technique, du bâtiment, de la guerre, de la loi, du milieu scolaire, de la domesticité, des arts, du tissu, du corps en général et de la santé en particulier, de l'animalité, des éléments, des conditions météorologiques, certains donnant lieu à des métaphores intraduisibles entre le français et l'anglais, de sorte que leur particularité culturelle ressort d'autant mieux ${ }^{28} .$. Par conséquent, du point de vue sémantique, l'unité paraitt s'instaurer moins au niveau du texte dans son ensemble qu'au niveau du paragraphe, voire de la phrase, degré quasi zéro de l'énoncé qui joue de la variété des images auxquelles il donne vie sur le papier comme dans notre imagination. Contrant de la sorte la lassitude qui pourrait être générée par l'effet de sérieux inhérent aux problèmes économiques, l'auteur utilise une technique de persuasion dont le rôle se rapproche de celui de l'humour quand il ne se confond pas avec lui : la métaphore opère une projection sur un autre plan, vecteur d'un décrochage vers l'imaginaire, nous invitant à suivre les lignes de fuite qui nous dévoilent d'autant mieux le tableau à considérer qu'elles nous en révèlent les multiples perspectives. Créateur d'images, fauteur de trouble au sens où il provoque une hésitation herméneutique entre littéral et figuré, abstraction et concrétude, le journaliste privilégie pour son argumentaire la temporalité de l'instant. Comme en un kaléidoscope, les métaphores conceptuelles, les métaphores linguistiques conventionnelles et les métaphores personnelles, qu'elles soient d'ordre textuel ou iconographique, se juxtaposent, se superposent ou s'opposent, pour nous offrir en couleurs ou en noir et blanc la représentation matérielle et immatérielle de tendances économiques nationales ou mondiales. À partir du factuel, la combinatoire de ces métaphores plurielles ouvre donc «les portes de la perception » sur la singularité d'une sensibilité, pour mieux nous faire entrer de plain-pied sur la scène économique.

\section{Conclusion}

Protéiforme ${ }^{29}$, au point de sembler relever de l'esthétique baroque en suscitant une anamorphose $\mathrm{e}^{30}$ dont elle paraît, de par sa nature même, être la figure-clé, la métaphore, comme l'a montré Paul Ricœur, est le lieu d'une tension vive entre le concret et l'abstrait, le littéral et le figuré. Elle incite le lecteur à dépasser l'opposition que scellent ces dernières antynomies ${ }^{31}$ pour qu'il les considère comme des vecteurs complémentaires dont la combinaison se révèle indispensable aux jeux des forces sémiotiques et à leurs enjeux pragmatiques. Loin d'être un ornement stylistique, elle décrit le lieu tantôt commun tantôt singulier où la réalité se laisse approcher depuis de multiples angles pour être conceptualisée en d'innombrables définitions que rythment l'union des 
ressemblances et la désunion des dissemblances. Au gré des traits saillants sélectionnés, cette figure qui prend forme linguistique ou visuelle, est, comme nous l'avons vu, très souvent à l'image du contexte culturel, notamment sportif, dans lequel elle apparaît : elle en est dès lors le signifiant tout en ne pouvant s'empêcher d'en être le signifié puisque le reflet qu'elle offre fait sens, éclairant à son tour d'une iconographie soit conventionnelle soit nouvelle un pan du réel qui la façonne ${ }^{32}$. Visuelle, la métaphore dont le fondement est fréquemment corporel, pointe l'espace qui sépare le connu de l'inconnu pour le fixer d'autant mieux qu'elle se veut unique, originale, créatrice, mobilisant les ressources de l'imagination pour instruire la raison. De là sa forte présence au fil des articles de journaux, y compris ceux de la presse spécialisée dans les domaines économique et politique, où elle saisit le lecteur à bras le corps-esprit, le frappe immédiatement, c'est-àdire instantanément et sans médiation, soit pour introduire le texte qui, dans son feuilleté, en saisira le grain, soit pour en fixer de façon synthétique un élément caractéristique. Dans le tourbillon des mots et des images - photographies, dessins, figures -, qui parfois se fondent, parfois se confondent quant à leur rôle respectif, elle s'associe aux métonymies et synecdoques pour jouer de thèmes universels et de variations nationales sur un mode qui lui est pourtant propre. L'instant d'une fulgurance, qui renvoie la dénotation à la connotation, les stéréotypes présentés et déconstruits cèdent la place à une indirection fondamentale qui diffracte les significations en instaurant la primauté du narratif sur le factuel. Elles s'enrichissent ainsi de la multiplicité des domaines où elles sont doublement réfléchies, au terme d'un jeu spéculaire entre le particulier et le général, le singulier et le pluriel, le même, l'autre et son double.

\section{Sources primaires anglaises ou américaines}

ADAMS Richard, "Latest polls show Romney and Obama locked in a tight race - US politics live. Mitt Romney and Barack Obama remain neck and neck in key swing states according to new polls", The Guardian, 8 Aug. 2012.

ANON., "An Olympic effort to retool the economy”, The Guardian, 12 Aug. 2012.

ANON., "As US and European economies sputter, investors hope for rescue by Fed, European Central Bank”, The Washington Post, 31 Jul. 2012.

ANON., "Blow for Obama as new figures show U.S. economy slowing even further as Americans cut back on spending", The Daily Mail, 27. Jul. 2012.

ANON., "Exports and the economy. Made in Britain. Exports are growing, but too slowly to rescue the economy”, The Economist, 21 Jan. 2012. 
ANON., "Jamaica at 50. On your marks, get set ...oh. Half a century after Jamaica's independence from Britain, its economy is struggling to get out of the starting blocks", The Economist, 2 Jul. 2012.

ANON., "Retail sales down in June for third straight month", bbc.co.uk, 16 Jul. 2012.

ANON., "The Spanish patient. A full bail-out of the euro area's fourth largest economy is looming", The Economist, 28 Jul. 2012.

ANON., "US economic growth slows in second quarter", bbc.co.uk, 27 Jul. 2012.

B.R., "Less for more", The Economist, 1 Aug. 2012.

DEWAN Shaila, « U.S. Economy Gains Steam as 200,000 Jobs Are Added”, The New York Times, 6 Jan. 2012.

DEWAN “U.S. Growth Slows to 2.2\%, Report Says", The New York Times, 27 Apr. 2012.

EL-ERIAN Mohamed, “Economics Blog”, The Guardian, 2 Aug. 2012.

FLANDERS Stephanie, “Could the US economy go the way of Japan?”, bbc.co.uk, 31 Aug. 2011.

HOPKINS Kathryn, "Everyone is going to be a winner, so let London's games begin", The Times, 3 Jul. 2012.

LYONS Gerard, "Economic Outlook: Lighten the load and Britain can win again", The Times, 29 Jul. 2012.

MARDELL Mark, “Is the US caught in the slow lane?”, bbc.co.uk, 5 Jul. 2012.

NORRIS Floyd, "U.S. Chose Better Path to Recovery", The New York Times, 3 May 2012.

RAMPLE Catherine, “Growth Accelerates, but U.S. Has Lots of Ground to Make Up”, The New York Times, 27 Jan. 2012.

RICH Motoko, “Job Gains Reflect Hope a Recovery Is Blooming”, The New York Times, 3 Feb. 2012.

SMITH David, “Economic Outlook: Use your muscle to boost lending, Guv'nor", The Times, 15 Jul. 2012.

TREANOR Jill \& MOULDS Josephine, “UK retailers fail to see boost from Olympic feelgood factor", The Guardian, 4 Sep. 2012.

WATTS Jonathan, FRANKLIN Jonathan \& BRODZINSKY Sibylla, "Latin America's booming economies face tough test. Ability to withstand the coming storm will have long-term implications for region that was until recently a byword for financial turbulence", The Guardian, 15 Aug. 2012.

\section{Sources primaires françaises}

C.P., « Paris ralentit la cadence mais le vert continue à dominer », Investir.fr, 1 août 2012.

DE MEYER Karl, « Malgré le ralentissement économique américain, la Fed opte pour le statu quo », Les Echos.fr, 2 août 2012.

ERNST Jonathan, « Obama s'attend à des “vents contraires” venus de la zone euro », Les Echos.fr, 31 juil. 2012.

PempEL Kacper, «La Maison blanche réduit ses prévisions de croissance », Les Echos.fr, 27 juil. 2012. 


\section{Sources secondaires. Ouvrages cités dans l'article}

Аміот Dany (Ed.), Études linguistiques. La métaphore : regards croisés, Journée d'études à Arras, mars 2002, Arras : Artois Presses Université, 2004.

ANONYME, Langage et pertinence : référence temporelle, anaphore, connecteurs et métaphore, Nancy : Collection Processus discursifs, Presses universitaires, 1994.

BUFFARD-MORET Brigitte, « La métaphore dans Les Fleurs du mal : Baudelaire, héritier du baroque », in AMIOT Dany (Ed.), Études linguistiques. La métaphore : regards croisés, Journée d'études à Arras, mars 2002, Arras : Artois Presses Université, 2004 : 35-50.

DEIGNAN Alice, Metaphor Dictionary, London: Harper-Collins, 1995.

GRÉA Philippe, « Logique de conformité et logique d'intégration », in AMIOT Dany (Ed.), Études linguistiques. La métaphore: regards croisés, Arras: Artois Presses Université, 2004: 101-122.

HAlliday Michael A. K. \& MATthiessen Christian M.I.M., Construing Experience through Meaning A Language-Based Approach to Cognition, London, New York: Continuum, 2006 [1999].

KÖVECSES Zoltan, Metaphor in Culture: Universality and Variation, Cambridge: Cambridge University Press, 2005.

LAKOFF George \& JOHNSON Mark, Metaphors We Live By, Chicago and London: The University of Chicago Press, 1980.

SYS Jacques, « La fonction métaphorique dans le Nouveau Testament », in АMIOT Dany (Ed.), Études Linguistiques. La métaphore: regards croisés, Arras: Artois Presses Université, 2004: 11-34.

WAY Eileen Cornell, Studies in Cognitive Systems Knowledge Representation and Metaphor, Dordrecht, Boston, London: Kluwer Academic Publishers, 1991.

\section{Sources secondaires. Articles parus dans des périodiques}

BRANDT Per Aage et Line, "Making sense of a blend: A cognitive-semiotic approach to metaphor", Annual Review of Cognitive Linguistics, vol. 3, No. 1, 2005: 216-249.

CAMERON Lynne, "Confrontation or Complementarity? Metaphor in Language Use and Cognitive Metaphor Theory”, Annual Review of Cognitive Linguistics, vol. 5, No. 1: 107-135.

DOBRIC Nikola, "Theory of names and cognitive linguistics - The case of the metaphor", Filozofija $i$ Društvo, vol. 21, No.1, 2010: 135.

HART Elizabeth, “Cognitive linguistics: the experiential dynamics of metaphor”, Mosaic, Winnipeg, vol. 28, No. 1, Mar. 1995: 1-23.

KÖVECSES Zoltan, "Conceptual metaphor theory Some criticisms and alternative proposals", Annual Review of Cognitive Linguistics, vol. 6, No.1, 2008: 168-184.

KÖVECSES Zoltan, “A new look at metaphorical creativity in cognitive linguistics”, Cognitive Linguistics, vol. 21, No. 4, Nov. 2010: 663-697.

STEEN Gerard J., "Identifying Metaphor in Language: a Cognitive Approach”, Style, vol. 36, No. 3, Fall 2002: 386-409. 
TURNER Mark \& FAUCONNIER Gilles, “A Mechanism of Creativity”, Poetics Today, vol. 20, No. 3, Fall

1999: 397-418.

\section{NOTES}

1. Cf. Le dictionnaire Robert : «Contexte » vient de « contextus » : assemblage, de « contexere »: tisser avec.

2. Date des JO 2012 de Londres : du 27 juillet au 12 août.

3. Cf. Kövecses Zoltan, Metaphor in Culture. Universality and Variation, Cambridge: Cambridge University Press, 2005: 95, où il est question de la dimension stylistique: "By style I mean linguistic variation according to the communicative setting, subject matter, medium, audience and other factors. For example, in newspaper articles about American football games, the names of the teams may inspire the selection of particular metaphors for defeat and victory ». Ici, l'élan est le même bien que la configuration soit différente puisqu'il s'agit d'importer des images d'un domaine vers un autre.

4. Cf. Kövecses [2005: 120] : la métaphore conceptuelle qu'il relève à propos de la politique ( POLITICS IS SPORT) vaut également pour l'économie.

5. Cela revient à noter l'importance de la prise en compte du contexte culturel. Cf. Kövecses [2005: 131] : « Four issues are investigated in some detail: [...] (4) how particular cultural contexts in which conceptual metaphors are embedded influence the linguistic expression of these metaphors ".

6. Celle-ci constitue une spécificité culturelle française. On pourra se reporter par exemple à l'article de C.P. : « Paris ralentit la cadence mais le vert continue à dominer », Investir.fr, $1^{\mathrm{er}}$ août 2012, où elle est filée d'un paragraphe à l'autre, et ce, dès le début : « La Bourse de Paris ralentit la cadence mais se maintient dans le vert. [...] Au chapitre des entreprises, EADS est en tête du Cac 40, tandis que Vinci est en queue de peloton. » Dates du Tour 2012 : du 30 juin au 22 juillet.

7. Kövecses [2005: 107] : «The creative use of metaphor has a great deal to do with individual variation in metaphor. Individuals often have experiences that do not conform to conventional patterns captured by conventional conceptual metaphors. A part of the creative genius lies in the ability to extend the range of particular target domains ». George Lakoff, Mark Johnson, Metaphors We Live By, Chicago, London: The University of Chicago Press, 1980: 53 : « instances of novel metaphor, that is, a metaphor not used to structure part of our normal conceptual system but as a new way of thinking about something ». Cf. Lynne Cameron, "Confrontation or Complementarity?: Metaphor in Language Use and Cognitive Metaphor Theory », Annual Review of Cognitive Linguistics, Vol. 5, No. 1: 116: « Clear examples of novel metaphor that are highly likely to involve active processing produce necessary conditions for metaphor identification: (1) the presence of lexical items from disparate (or incongruent) semantic fields and (2) the potential for extra meaning to be produced as a result of bringing these together ».

8. À rapprocher d'une des métaphores conceptuelles récurrentes de la politique américaine, cf. Kövecses [2005: 187] : «there can be no doubt that in America Politics (and especially the ELECTIONS) IS A SHOW ».

9. Kövecses [2005: 17] : «It is a well-known fact that people around the world conceptualize time in terms of space (Alverson, 1994)».

10. Deux métaphores conceptuelles prévalent pour la représentation du temps: TIME IS HORIZONTAL et TIME IS VERTICAL. Cf. Kövecses [2005: 30, 51, 53]: "Another major way to conceptualize time metaphorically is the MOVING OBSERVER metaphor ». [...] « Time and motion are literally correlated in basic ways. The literal correlation joins time as a domain and motion as a domain in a single literal conceptual frame, or schema, the TIME-MOTION frame. Given that literal 
frame, elements of the two frames can stand metonymically for each other within the single unified frame. This can show up in the form of linguistic metonymies. For instance, in English one can say, "I slept for fifty miles while she drove" (DISTANCE FOR TIME-DURATION) 》.

11. Cf. Karl de Meyer, « Malgré le ralentissement économique américain, la Fed opte pour le statu quo ", Les Échos.fr, 2 août 2012, 1. 1 : « La banque centrale laisse le Federal Funds Rate dans son couloir de 0 à $0,25 \%$ ».

12. Kövecses [2005: 15]: «In cognitive linguistics, metaphor is a set of conceptual correspondences, or more technically, mappings, between two conceptual domains, a source and a target (Lakoff and Johnson, 1980 ; Kövecses, 2002). The correspondences between a source and a target domain make up a conceptual metaphor ». Cf. Halliday Michael A. K., Matthiessen Christian M.I.M., Construing Experience through Meaning A Language-Based Approach to Cognition, London, New York : Continuum, 2006 [1999] : 7: «grammatical metaphor [...] is a phenomenon whereby a set of agnate (related) forms is present in the language having different mappings between the semantic and the grammatical categories.»

13. Cf. Lakoff \& Johnson [1980]. Kövecses [2005 : 43] : «The Event Structure Metaphor. In this metaphor, different aspects of events, such as state, change, cause, action, and purpose, are comprehended via a small set of physical concepts: location (bounded region), force and movement »; [2005:47] : «the submappings of this metaphor complex are all simple or primary metaphors that are motivated independently of each other. For example, [...] movement leads to change of location; [...] if we want to achieve a purpose, we often have to move to particular destinations ; sometimes we have to make choices among the paths that lead to destinations to achieve our goals. These correlations in bodily experience provide independent motivation for each of these submappings, and they jointly motivate the existence of the Event Structure Metaphor ».

14. À rapprocher des métaphores relevées par Lakoff et Johnson $[1992,1996]$ pour la politique américaine et citées par Kövecses [2005: 174] : « THE PRESIDENTIAL ELECTION IS A RACE ». Lakoff et Johnson [1980: 31] : «A race [...] is an event, which is viewed as a discrete entity. The race exists in space and time, and it has well-defined boundaries. Hence we view it as a CONTAINER OBJECT, having in it participants (which are objects), events like the start and finish (which are metaphorical objects), and the activity of running (which is a metaphorical substance) ». À titre d'exemple, on pourra se reporter à l'article de Richard Adams paru dans The Guardian le 8 août 2012 et intitulé « Latest polls show Romney and Obama locked in a tight race - US politics live. Mitt Romney and Barack Obama remain neck and neck in key swing states according to new polls ».

15. Mark Turner, Gilles Fauconnier, "A Mechanism of Creativity », Poetics Today, Vol. 20, No. 3, Fall 1999: 405 : «A flag is a symbol - for example, fifty stars for fifty states. Understanding a nation as a physical object [...] does not delude us into believing that the physical flag is actually the nation it represents, but blending may be at work in this metaphor. [...] the flag may count as more than mere representation. In the blend, an attack on the flag is an attack on the nation. We are not trapped inside blends, but emotional reactions generated there can, like inferences generated there, leave their mark on reality ".

16. Incarnation qui est comme un rappel du fondement corporel des métaphores (Cf. Kövecses [2005: 2 ] : "According to the "standard" view of metaphor in the Lakoff-Johnson framework, metaphors are based on embodied human experiences ») et qui est basée sur la métaphore conceptuelle A STATE IS A PERSON (cf. Kövecses [2005: 174]).

17. Kövecses [2005: 153] : «the degree of conventionalization is the extent of stylistic marking or nonmarking (that is, stylistic neutrality) particular metaphorical expressions have (Barcelona, 2001)».

18. Relation fondée sur la structure événementielle PROGRESS IS MOTION FORWARD. Pour un autre exemple de cette dernière, on pourra se reporter à Kövecses [2005: 237]. 
19. Image des entraves que l'on retrouve, mais inversée, dans l'article de Shaila Dewan, «U.S. economy Gains Steam as 200,000 Jobs Are Added», The New York Times, 6 Jan. 2012, 1. 64 : « This is finally the economy throwing off the shackles of the credit crunch » et dans « Exports and the economy. Made in Britain. Exports are growing, but too slowly to rescue the economy ", The Economist, 21 Jan. 2012, 1. 29 : « the euro zone will contract by $1.2 \%$ in 2012. One Conservative MP, Douglas Carswell, has complained that it is like being 'shackled to a corpse' ».

20. Lakoff \& Johnson [1980: 36-37] : « metaphor is principally a way of conceiving of one thing in terms of another, and its primary function is understanding. Metonymy, on the other hand, has primarily a referential function, that is, it allows us to use one entity to stand for another. But metonymy is not merely a referential device. It also serves the function of providing understanding. [...] it allows us to focus more specifically on certain aspects of what is being referred to. It is also like metaphor in that it is not just a poetic or rhetorical device ».

21. Lakoff \& Johnson [1980: 14] : « in some cultures the future is in front of us, whereas in others it is in back»; [1980: 42] : «time in English is structured in terms of the TIME IS A MOVING OBJECT metaphor, with the future moving toward us ».

22. Pour une définition générale des métaphores ontologiques, cf. Lakoff \& Johnson [1980: 26, 33-36].

23. Pour une étude des théories qui établissent un lien entre métaphore et émotion, on pourra se reporter à Eileen Cornell Way, Studies in Cognitive Systems Knowledge Representation and Metaphor, Dordrecht, Boston, London: Kluwer Academic Publishers, 1991: 30: «The major theories on metaphor that I will discuss here are emotive, substitution, anomaly and interactive theories. Emotive theories see the role of metaphor in language to be purely emotional and lacking in any cognitive content ».

24. Cf. Kövecses [2005: 239] : « we can expect an increase in the relative salience of the ECONOMY IS HEALTH metaphor in the winter period (Boers, 1999)» et cf. Kövecses [2008: 179]. Cf. Nikola Dobrić, "Theory of names and cognitive linguistics - The case of the metaphor ", Filozofija $i$ Društvo, Vol.21, No. 1, 2010: 140: "[in the case of] the metaphorical concept connected to political discourse ECONOMY IS A BODY - to put the economy back on its feet; the recovery of the economy; to strengthen the economy; etc. the mental connection between target domain of ECONOMY and the source domain of BODY is not so obvious ».

25. Elizabeth Hart, «Cognitive Linguistics: The Experiential Dynamics of Metaphor», Mosaic, Vol. 28, No. 1, Mar. 1995: 20 : «the pun is a problem of metaphor, Ferguson describes its effect as "a divorce between words and their conventional meanings. The split between sign and signified [...] leads to a materializing [of] the word, [...] that forces us to question the distinction between literal and figurative meanings" ".

26. Philippe Gréa, "Logique de conformité et logique d'intégration», in Amiot Dany, Études linguistiques. La métaphore: regards croisés, Arras: Artois Presses Université, 2004: 119 : «la métaphore filée [...] acquiert un statut théorique autonome puisqu'elle est le lieu d'un mécanisme qui lui est propre [et] se caractérise par son extrême modifiabilité [...] l'intégration conceptuelle est un processus fondé sur un système de contraintes suffisamment souple pour rendre compte d'une multitude de variations thématiques, tout en permettant une mesure de la cohérence (ou de l'optimalité) du réseau d'intégration ».

27. Sur les « mixed metaphors », Cf. Lakoff \& Johnson [1980: 94-7].

28. Cet obstacle de traduction se signale notamment par des guillemets, comme dans l'article de Jonathan Ernst, «Obama s'attend à des "vents contraires" venus de la zone euro ", Les Échos.fr, 31 juillet 2012, 1. 1 : «Barack Obama a prévenu lundi que l'économie américaine allait continuer à subir des "vents contraires" dans les prochains mois sous l'effet de la crise de la dette de la zone euro tout en se montrant confiant sur la survie de la monnaie unique » ou dans celui de Kacper Pempel, «La Maison blanche réduit ses prévisions de croissance », Les Échos.fr, 27 juil. 2012, 1. 9 : «"L'économie continue d'affronter d'importants vents contraires [...] explique le rapport sur le 
projet de budget 2013 » ou l. 25 : «UN "MUR BUDGETAIRE EN VUE ». L'image du navire dans la tempête qui doit faire face à ces vents appelés « headwinds » semble propre à l'anglais, de même que celle du mur auquel on se heurte pour évoquer de très graves problèmes budgétaires. L'obstacle traductologique peut aussi être signalé par un adjectif comme «fameux/-se » qui fait appel aux connaissances partagées, comme dans l'article de C.P. , «Paris ralentit la cadence mais le vert continue à dominer ", Investir.fr, 1 août 2012, 1. 13- 14 : « et ce alors que la fin de l'année sera marquée par les élections présidentielles et la fameuse falaise fiscale », ou un participe passé employé comme adjectif, tel «surnommé/-e », dans l'article de K. Pempel. 1. 27 : « une échéance surnommée le "mur budgétaire" ".

29. Sur le lien entre métaphore et anamorphose, on pourra se reporter à l'article de Jacques Sys, in Amiot Dany, Études Linguistiques La métaphore : regards croisés [2004 : 27].

30. Sur le lien entre métaphore et baroque, on pourra se reporter à l'article de Brigitte BuffardMoret, in Amiot Dany, Études linguistiques La métaphore : regards croisés [2004 : 38-42].

31. Elizabeth Hart, Mosaic, Vol. 28, No.1, Mar. 1995: 9, évoque un «continuum»: "the experiential model recasts this difference [between literal and metaphorical], however, not as essential or as binary opposition, but as two potentially simultaneous stages of semantic activity taking place within the same continually shifting system - a system that situates the concrete and the abstract on a continuum with, rather than in opposition to, each other ».

32. Cf. Gerard J. Steen, «Identifying Metaphor in Language: a Cognitive Approach », Style, Vol. 36, No. 3, Fall 2002: 403: «What I am about to suggest here is rather speculative, but it may help to model how meaning, including metaphorical meaning gets incrementally produced ».

\section{RÉSUMÉS}

Cet article se propose d'étudier l'influence du contexte international en général, et des événements récurrents comme les Jeux Olympiques en particulier, sur la création de métaphores en prise directe avec l'actualité dans les domaines politique et économique. Le corpus est constitué d'une trentaine de textes de la presse britannique et américaine contemporaine, dont les idiomatismes révélateurs de spécificités culturelles sont mis en lumière au travers de comparaisons avec des extraits de la presse française sur les mêmes thèmes, dont celui de la crise globale et de ses répercussions aux États-Unis, en Europe, notamment en Grande-Bretagne, et en Jamaïque. La ligne directrice de cette analyse, qui a pour cadre théorique la linguistique cognitive et son approche de la métaphore conceptuelle, vise à dégager des invariants dans l'émergence de la métaphore tant textuelle que visuelle pour mieux révéler les variations auxquelles elle se prête en donnant d'emblée à entendre, et parfois à voir, l'un des contrepoints du discours littéral.

This paper focuses on the role played by the international context in general, and by such recurring events as the Olympic Games in particular, in the emergence of topical political and economic metaphors. The study of thirty-odd contemporary newspaper articles from the British and American press allows us to highlight the idioms that are coloured by the cultural characteristics of each country thanks to the three-way comparison with French newspaper articles on the same themes, including that of the current global crisis and its effects across the US, Great-Britain, Europe and Jamaica. The guiding line of this analysis, whose framework is provided by the Conceptual Metaphor Theory of the American school of cognitive linguistics, aims to point to invariants in emerging textual or visual metaphors better to underline the 
variations the latter are subject to, while letting themselves be perceived at once as so many counterpoints to any literal discourse.

INDEX

Mots-clés : actualité, économie, politique, contexte international, Jeux Olympiques

Keywords : topicality, economics, politics, the international context, Olympic Games

\section{AUTEUR}

CATHY PARC

Institut Catholique de Paris, France

c.parc@yahoo.fr 